\title{
Revisión taxonómica del grupo Haplothrips-Karnyothrips (Thysanoptera: Phlaeothripidae)
}

\author{
Axel P. Retana-Salazar ${ }^{1} \&$ Gerardo A. Soto-Rodríguez ${ }^{2}$ \\ 1 Centro de Investigación en Estructuras Microscópicas (CIEMic), Ciudad de la Investigación, Universidad de Costa \\ Rica; 2060, San José, Costa Rica; apretana@cariari.ucr.ac.cr \\ 2 Hypericum Pharma, S.A. Apartado 1237-2050 San Pedro MO, San José, Costa Rica; hiperiam@amnet.co.cr
}

Recibido 30-XI-2004. Corregido 24-I-2007. Aceptado 23-II-2007.

\begin{abstract}
Taxonomic revision of the Haplothrips-Karnyothrips group (Thysanoptera: Phlaeothripidae). The generic group ("ensemble") Haplothrips-Karnyothrips is reviewed, following the most recently published criteria for distinguishing generic characters. We establish a new diagnosis for each genus. A review of a Central American collection is included and a new genus of Phlaeothripidae is described from the Central Pacific of Costa Rica from specimens collected on Cyperaceae flowers during the dry season. The genus can be distinguished by widely separated maxillary stylets, absent maxillary bridge, pelta shape and the setae B1, which measures two thirds of the tube length. We include a key based on characters of phylogenetic importance. Rev. Biol. Trop. 55 (2): 627-635. Epub 2007 Jun 29.
\end{abstract}

Key words: Phlaeothripinae, Thysanoptera, new genus, Costa Rica.

Los "trisanópteros" son insectos cosmopolitas que se encuentran distribuidos por todo el mundo; la mayoría de las especies se encuentran en el trópico y unas pocas en las regiones árticas. Sus hábitat incluyen bosques, pastizales, desiertos, tierras cultivadas, jardines (Lewis 1973, Mound 2002).

Dentro de estos, en el suborden Tubulifera, la subfamilia Phlaeothripinae incluye más de 2500 especies, unas pocas son depredadoras, otras son fitófagas y forman agallas en sus hospederos y otras se alimentan de hifas de hongos en madera muerta o en hojarasca (Mound 1997).

Se distinguen por poseer estiletes maxilares más cortos que los Idolothripinae (la otra subfamilia de Tubulifera), los machos presentan un área glandular en el segmento abdominal VIII y la seta B2 del tergito IX es corta y ancha (Mound y Marullo 1996; Goldarazena y Mound 1998).

En general, los hábitat en que se desarrollan estas especies incluyen restos vegetales tales como ramas con follaje seco o muerto, árboles o ramas sobre el suelo, hojarasca, musgos y líquenes (Ananthakrishnan 1984). Condiciones de humedad y de temperatura adecuados en estos hábitat favorecen el desarrollo de hongos saprófitos que luego constituyen el alimento de estos insectos, sobre todo en las etapas tempranas de desarrollo del hongo. Este tipo de alimentación proporciona una fuente importante de energía y parece estar relacionada con los requerimientos de ergosterol que se encuentran contenidos en los hongos, los cuales permiten a las poblaciones de "thrips" sobrevivir por períodos de tiempo más prolongados (Ananthakrishnan 1984).

En este trabajo se revisa el grupo Haplohrips y se aclaran las relaciones dentro del género parafilético Karnyothrips, y se describe un nuevo género del Neotrópico procedente del Pacífico de Costa Rica, se separan además tres nuevos géneros con criterios filogenéticos. Se acompaña de una clave actualizada para este conjunto de géneros emparentados o "ensemble". 


\section{MATERIAL Y MÉTODOS}

Se examinó material recolectado en la provincia de Puntarenas, Costa Rica. Los especímenes fueron tratados con $\mathrm{NaOH}$ y montados en bálsamo de Canadá. Las observaciones, dibujos y medidas respectivas se realizaron con un microscopio de luz polarizada provisto de micrómetro a 100x y 400x.

El material se encuentra depositado en la colección de Thysanoptera del primer autor de este trabajo la cual se halla en Museo de Insectos de la Universidad de Costa Rica (MIUCR). Se revisaron especímenes de los géneros Haplothrips Amyot y Serville 1843 y Karnyothrips Watson 1924, depositados en la misma colección.

Análisis de caracteres del grupo Haplothrips: El análisis de caracteres demuestra que utilizando los caracteres habituales definidos por los taxónomos $\alpha$ para la separación de grupos genéricos, existe una enorme variación entre los caracteres que convierten a los géneros en grupos mal definidos, aplicando la conceptualización expuesta por Retana-Salazar y Retana-Salazar (2004) la determinación de los linajes en función de la relación c-inclusión de los caracteres se obtiene una serie de caracteres (Cuadro 1) donde los únicos estables para definir linajes genéricos son: a) los conos sensoriales en el antenómero IV, donde la presencia de varios sensores en el segmento IV es una condición derivada (Mound et al. 1980), que justifica su utilización en la determinación de grupos (Retana-Salazar 1998, 2000, RetanaSalazar y Soto-Rodríguez 2005) b) el desarrollo de puente maxilar y c) la basantra o mejor denominada placas del praepectum.

Desde un punto de vista filogenético por la historia evolutiva asociada a la obtención de estructuras complejas como los conos sensoriales y como lo señala Mound y colaboradores (1980) por su estado de derivación en cuanto al número de estas estructuras, son de trascendental importancia en la definición de linajes. Como se observa los conos asociados al antenómero III son altamente variables, por
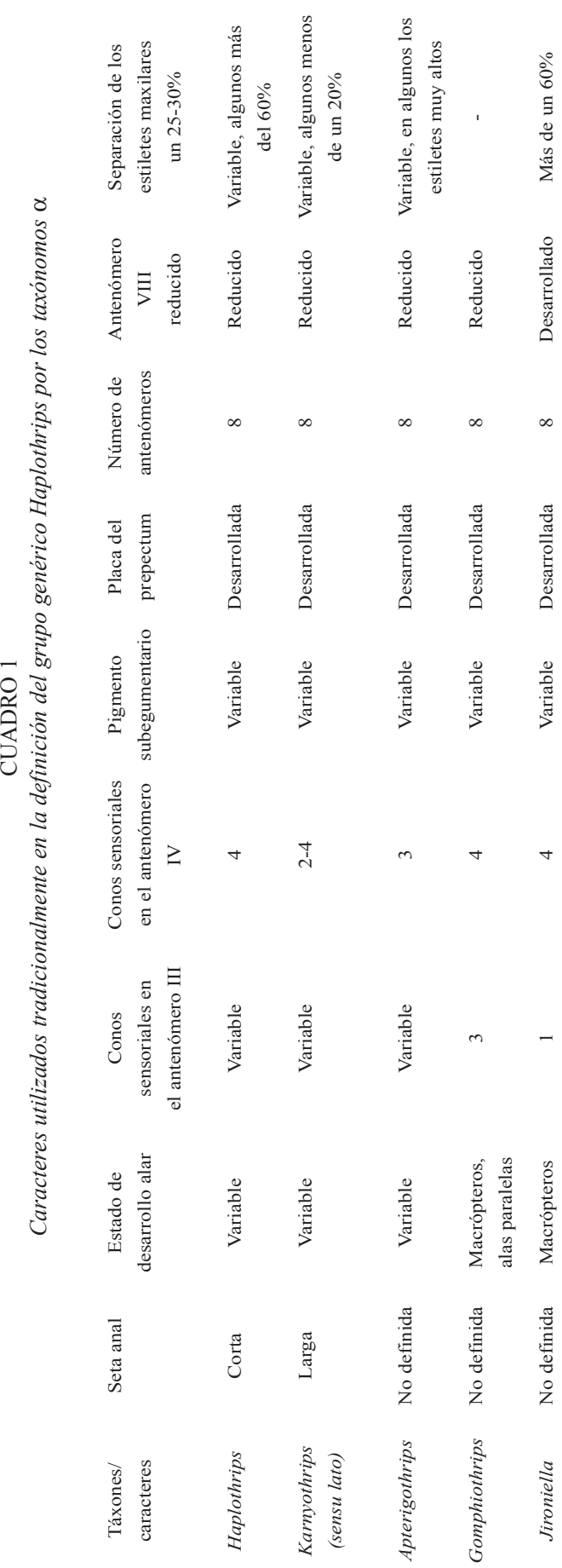

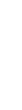


lo que son importantes en la definición de especies pero no es útil en la definición de linajes supraespecíficos, por el contrario, la presencia de conos sensoriales en el antenómero IV varía entre grupos genéricos excepto en el caso de Karnyothrips que, donde después de un análisis detallado se concluye que se trata de un género parafilético que debe ser separado para una mejor comprensión de sus relaciones, razón por la que se revisa el grupo Karnyothrips, pero merecen igual atención Haplothrips, Apterygothrips y Adraneothrips.

Con respecto al desarrollo del tentorium, Mound y colaboradores (1980) definen, que en los phlaeothrípidos existe una condición de amplia reducción del tentorium, presentando únicamente las bases de los brazos anteriores. Con respecto a esto es complicado definir si la presencia de un puente maxilar es a) una adquisición secundaria y en consecuencia una apomorfía, o b) la pérdida de esta estructura debe considerarse como apomórfica. Lo expuesto por Mound y colaboradores implicaría que la presencia de puente maxilar es una derivación secundaria, sin embargo hemos sometido a prueba la hipótesis examinando tanto de la literatura como los especímenes de museo con el fin de determinar si al menos en este grupo constituido para nosotros por los géneros Gomphiothrips, Karnyothrips (sensu lato), Apterygothrips, Haplothrips y el nuevo género Jironiella si el puente maxilar es una estructura que se mantiene o no, logrando determinar que es posible que el estado plesiomórfico lo constituya la presencia de esta estructura y en consecuencia su pérdida en Jironiella es una apomorfía del nuevo grupo genérico.

Grupo Karnyothrips: Al analizar los caracteres se concluye que el único género que rompe la continuidad de los caracteres es Karnyothrips, evidenciando un agrupamiento parafilético, que debe ser esclarecido. Se hallan varios táxones unidos bajo este nombre, por consideraciones morfológicas y no evolutivas, ya que las especies de este género no son homogéneas en sus caracteres fundamentales lo que justifica su separación e impide una correcta delimitación del género Karnyothrips. Esto se refleja en la última clave genérica presentada por Mound y Marullo (1996) donde los autores no hacen una diferenciación entre el género Karnyothrips y Apterygothrips.

Para esto los autores han decidido seguir los lineamientos para la separación de táxones postulados por Retana-Salazar y RetanaSalazar (2004). Con los postulados lógicos de estos autores se pretende que los nuevos táxones adquieran un mayor fundamento filogenético y biológico, de esta forma nos aproximamos a una clasificación natural.

Tomando en cuenta que el género Karnyothrips en estricto sentido estaría conformado por las especies con 4 conos sensoriales en el antenómero IV y con setas am reducidas, ya que la especie tipo del género Karnyothrips flavipes (=Karynia weigeli Watson es sinónimo de Anthothrips flavipes Jones, nombre que se mantiene en el actual género Karnyothrips) muestra este conjunto de caracteres, en conjunto con esta especie se halla la especie merilli descrita por Watson en 1920 para el género Haplothrips, luego transferida a Karnyothrips. En consecuencia el género Karnyothrips consta de tan solo dos especies, el resto de las especies con 3 conos sensoriales en el antenómero IV y setas am reducidas pasan a conformar el nuevo género Vargasia gen. n., mientras que las especies con 3 conos sensoriales en el antenómero IV y setas am desarrolladas conforman el nuevo género Willeia gen. n. (cuadros 2 y 3). Una especie de difícil ubicación resulta ser sonorenis la cual exhibe tan solo 2 conos sensoriales en el antenómero IV y el desarrollo de las setas am. Se ha tomado en consideración que su ubicación dentro de cualquiera de estos géneros rompería la solidez del caracter estudiado y ante la ausencia de evidencia que permita incluirla en cualquiera de estos agrupamientos sin romper la unidad evolutiva que parecen establecer se traslada al nuevo género Dioclesianothrips gen. n. (cuadro 3).

Con el fin de estimar la validez de la hipótesis planteada se efectúa un análisis cladista de este grupo incluyendo al género Jironiella. Par esto se utilizaron los 11 caracteres que 
CUADRO 2

Segregación del grupo Karnyothrips en función de los conos sensoriales del antenómero IV y el desarrollo de las setas am del pronoto

\begin{tabular}{|c|c|c|c|}
\hline $\begin{array}{l}\text { Listado de especies de } \\
\text { Karnyothrips del Nuevo Mundo }\end{array}$ & $\begin{array}{l}\text { Ubicación genérica de cada especie } \\
\text { según Johansen \& Mojica (1993) }\end{array}$ & $\begin{array}{l}\text { Ubicación genérica de cada especie } \\
\text { según Mound \& Marullo (1996) }\end{array}$ & $\begin{array}{l}\text { Ubicación genérica de cada especie } \\
\text { según Retana \& Soto (2004) }\end{array}$ \\
\hline flavipes Jones & Karnyothrips & Karnyothrips & Karnyothrips \\
\hline merrilli Watson & Karnyothrips & Karnyothrips & Karnyothrips \\
\hline americanus Hood n. comb. & Karnyothrips & Karnyothrips & Willeia gen. n. \\
\hline rhopalocerus Hood n. comb. & Karnyothrips & Karnyothrips & Willeia gen. n. \\
\hline sonorensis Stannard n. comb. & Karnyothrips & Karnyothrips & Dioclesianothrips gen. $\mathrm{n}$. \\
\hline analis De Santis n. comb. & Adraneothrips & Karnyothrips & Vargasia gen. $\mathrm{n}$. \\
\hline antennatus Hood n. comb. & Karnyothrips & Karnyothrips & Vargasia gen. $\mathrm{n}$. \\
\hline anthracinus Hood n. comb. & Karnyothrips & Karnyothrips & Vargasia gen. n. \\
\hline arizona Hood n. comb & Karnyothrips & Karnyothrips & Vargasia gen. n. \\
\hline brimleyi Hood stat. $\mathrm{n}$. & Karnyothrips & Karnyothrips & Vargasia gen. n. \\
\hline bromelianus Johansen & Apterigothrips & Karnyothrips & Apterigothrips \\
\hline caliginosus Hood n. comb. & Karnyothrips & Karnyothrips & Vargasia gen. $\mathrm{n}$. \\
\hline caxamarca Hood n. comb. & Karnyothrips & Karnyothrips & Vargasia gen. n. \\
\hline dodgei Hood n. comb. & Karnyothrips & Karnyothrips & Vargasia gen. n. \\
\hline festivus Hood n. comb. & Karnyothrips & Karnyothrips & Vargasia gen. $\mathrm{n}$. \\
\hline franciscanus Hood n. comb. & Karnyothrips & Karnyothrips & Vargasia gen. $\mathrm{n}$. \\
\hline harti Hood n. comb. & Karnyothrips & Karnyothrips & Vargasia gen. $\mathrm{n}$. \\
\hline longiceps Hood & Apterigothrips & Karnyothrips & Apeterigothrips \\
\hline medialis Hood n. comb. & Karnyothrips & Karnyothrips & Vargasia gen. $\mathrm{n}$. \\
\hline melaleucus Baganall n. comb. & Karnyothrips & Karnyothrips & Vargasia gen. n. \\
\hline minimus Johansen n. comb. & Karnyothrips & Karnyothrips & Vargasia gen. n. \\
\hline noveboracensis Hood n. comb. & Karnyothrips & Karnyothrips & Vargasia gen. n. \\
\hline ochropezus Hood n. comb. & Karnyothrips & Karnyothrips & Vargasia gen. n. \\
\hline piceus Hood n. comb. & Karnyothrips & Karnyothrips & Vargasia gen. n. \\
\hline politus Johansen n. comb. & Karnyothrips & Karnyothrips & Vargasia gen. n. \\
\hline prolatus Hood n. comb. & Karnyothrips & Karnyothrips & Vargasia gen. $\mathrm{n}$. \\
\hline sympathicus Johansen n. comb. & Karnyothrips & Karnyothrips & Vargasia gen. $\mathrm{n}$. \\
\hline $\begin{array}{l}\text { tepoztlanensis Johansen \& Mojica } \\
\text { n. comb. }\end{array}$ & Karnyothrips & Karnyothrips & Vargasia gen. n. \\
\hline texensis Hood stat. n. & Karnyothrips & Karnyothrips & Vargasia gen. n. \\
\hline venustus Moulton n. comb. & Karnyothrips & Karnyothrips & Vargasia gen. $\mathrm{n}$. \\
\hline
\end{tabular}

se consideran informativos desde el punto de vista evolutivo.

1) Seta anal: larga 1 , corta 0,2$)$ Ala: bien desarrollda 1 , poco desarrollada 0,3$) 4$ sensores en IV 1 , diferente 0,4$) 3$ sensores en IV 1 , diferente 0,5$) 2$ sensores en IV 1 , diferente 0 ,
6) Antenómero VIII: reducido 1, bien desarrollado 0,7) Puente maxilar: presente 1, ausente $0,8)$ Setas am cortas 1, diferente 0, 9) Setas am largas 1 , diferente 0,10 ) setas pa cortas 1 , diferente 0,11 ) setas pa largas 1 , diferentes 0 (apéndice 1) (Fig. 4). 
CUADRO 3

Caracteres que definen a cada uno de los géneros del grupo Karnyothrips Watson 1924

\begin{tabular}{lcc}
\multicolumn{1}{c}{ Taxa } & $\begin{array}{c}\text { Conos sensoriales } \\
\text { en el antenómero }\end{array}$ & $\begin{array}{c}\text { Quetotaxia } \\
\text { del pronoto }\end{array}$ \\
IV & 4 & Setas am pequeñas \\
Karnyothrips & 3 & Setas am pequeñas \\
Vargasia gen. n. & 3 & Setas am=aa largas \\
Willeia gen. n. & 2 & Setas am $=$ aa largas \\
Dioclesianothrips gen. n. & &
\end{tabular}

Grupo Jironiella: De estos géneros el único que no presenta puente maxilar es el nuevo taxón Jironiella, esto nos indica que debe ubicarse en un nuevo grupo genérico ya que no cumple con el postulado básico necesario para la agrupación de táxones de cumplir con la relación estricta de c-inclusión, con respecto a los caracteres definitorios de los grupos (Retana-Salazar y Retana-Salazar 2004).

Este grupo consta por el momento de un solo taxón con una sola especie, caracterizado por la ausencia de puente maxilar, los estiletes maxilares se hallan por debajo de los ojos sin llegar a la mitad de la cabeza, mientras que se hallan separados por más de un $33 \%$ del ancho de la cabeza, hay reducción de las setas del pronoto y presenta 4 conos sensoriales en el IV antenómero.

En este estudio no incluimos a Hexagoniothrips por no compartir el criterio de Mound y Marullo (1996) considerando sus especies como sinónimas de otros grupos. Creemos indispensable un análisis más profundo de estas especies y sus caracteres antes de aceptar cambios serios en la taxonomía del grupo.

\section{Karnyothrips}

\section{Watson 1924}

Especie tipo: Karnyothrips flavipes Jones 1912.

Diagnosis: Puente maxilar presente, antenómero IV con 4 conos sensoriales, antenómero III variable en el número de conos sensoriales, quetotaxia del pronoto con las setas am reducidas con relación a las setas aa.

Material: especímenes de Karnyothrips merilli de la colección de A.P. Retana-Salazar

Número de especies incluidas: 2, flavipes y merrilli.

Comentario: descrito por Watson en 1922 bajo el nombre de Karynia y cambiado a Karnyothrips en 1924 por Watson. La especie tipo fue Karynia weigeli Watson, sinonimia de Anthothrips flavipes Jones 1912. El género desde sus inicios ha sido complicado, esto posiblemente por caracteres morfológicos superficiales de fácil convergencia con otros grupos taxonómicos, lo cual enturbia la delimitación genérica. Debido a que la especie tipo del género cuenta con 4 conos sensoriales en el antenómero IV, sólo las especies con esta característica pueden ser consideradas como Karnyothrips aunque estas constituyan la excepción dentro del género que fue equivocadamente descrito con 3 conos sensoriales en el antenómero IV sin considerar las condiciones de la especie tipo. En consideración de lo explicado anteriormente acerca de los caracteres con importancia biológica y filogenética se procede a la separación del grupo.

\section{Vargasia gen. n.}

\section{Retana \& Soto}

Especie tipo: Karnyothrips melaleucus Bagnall 1911, por designación.

Diagnosis: Puente maxilar presente, antenómero IV con 3 conos sensoriales, antenómero III con número variable de conos sensoriales, quetotaxia del pronoto con las setas am reducidas con relación a las setas aa.

Material: especímenes de Vargasia melaleuca, n.comb. y Vargasia ochropezus, n.comb. de la colección de A. P. Retana-Salazar

Número de especies incluidas: 24 especies. Todas transferidas del antiguo género Karnyothrips. Se mantienen las especies bromelianus y longiceps según la determinación de Johansen y Mojica (1993) en el género Apterygothrips hasta que no halla suficientes 
evidencias biológicas para traspasar estas especies al nuevo género Vargasia.

Etimología: Dedicado a José Antonio Vargas Zamora, uno de los más connotados especialistas en ecología tropical y en invertebrados marinos.

Comentario: El nuevo género Vargasia obedece a la segregación del género Kanyothrips utilizando caracteres de valor evolutivo, o de constancia morfológica comprobada, que permitan establecer con relativa facilidad los límites de los táxones supraespecíficos. Este nuevo género obedece a una separación fundamentada en criterios filogenéticos.

\section{Willeia gen. n.}

\section{Retana \& Soto}

Especie tipo: Karnyothrips americanus Hood 1912, por designación.

Diagnosis: Puente maxilar presente, antenómero IV con 3 conos sensoriales, antenómero III con número de conos sensoriales variable, quetotaxia del pronoto con las setas am bien desarrolladas y subiguales a las setas aa.

Número de especies incluidas: 2 especies. Las especies americanus y rhopalocerus pertenecen un grupo reducido de especies con las setas am desarrolladas. La especie sonorensis ha sido considerada aparte en función de los conos sensoriales del antenómero IV que es el caracter más estable y de peso filogenético en este grupo de géneros.

Etimología: Dedicada a Alvaro Wille Trejos, pionero de la entomología en Costa Rica y uno de los primeros entomólogos costarricenses en intentar análisis evolutivos.

Comentario: Dentro del género Karnyothrips se evidencian dos grandes grupos perfectamente delimitados por el desarrollo de la quetotaxia anterior del pronoto, la estabilidad de esta característica fundamenta la separación de dos grupos bien delimitados y excluyentes entre sí por los postulados de formación de linajes establecidos por RetanaSalazar y Retana-Salazar (2004), razón por la cual se ha considerado su separación.

\section{Dioclesianothrips gen. $\mathbf{n}$. Retana \& Soto}

Especie tipo: Karnyothrips sonorensis Stannard 1956a:25, por designación.

Diagnosis: Puente maxilar presente, antenómero IV con 2 conos sensoriales, antenómero III característico en forma, truncado apicalmente, antenómeros VII y VIII fuertemente unidos, setas am desarrolladas y subiguales a las setas aa.

Material: especímenes de la especie Dioclesianothrips sonorensis n.comb. de la colección de A.P.Retana-Salazar

Número de especies incluidas: 1, la especie sonorensis descrita por Stannard para el género Haplothrips, en el subgénero Xylaplothrips. La localidad tipo es California, hallándose también en Nuevo México, Utah, Idaho y Costa Rica.

Etimología: Dedicado a Dioclesiano por el apoyo brindado al primer autor hasta el último día de su vida.

Comentario: Aunque algunos taxónomos han incluido esta especie dentro de Karnyothrips en el grupo de especies con quetotaxia anterior del pronoto desarrollada junto con las especies americanus y rhopalocerus, este agrupamiento está justificado solamente por la convergencia de quetotaxia, pero obviando la diferencia en la composición de los conos sensoriales del antenómero IV donde solo se hallan 2 de estas estructuras. Se aprecia además una variación en la estructura del antenomero III y una unión fuerte entre los antenómeros VII y VIII, con lo que se considera como un conjunto de caracteres particulares que determinan la existencia de un linaje separado descrito aquí bajo el nombre de Dioclesianothrips.

\section{Jironiella gen. $\mathbf{n}$. \\ Retana \& Soto}

(Figs. 1-3)

Diagnosis: Cabeza ancha y del doble de longitud que el segmento abdominal X. Setas postoculares largas y de ápices ligeramente expandidos. Ocelo anterior ubicado en una 

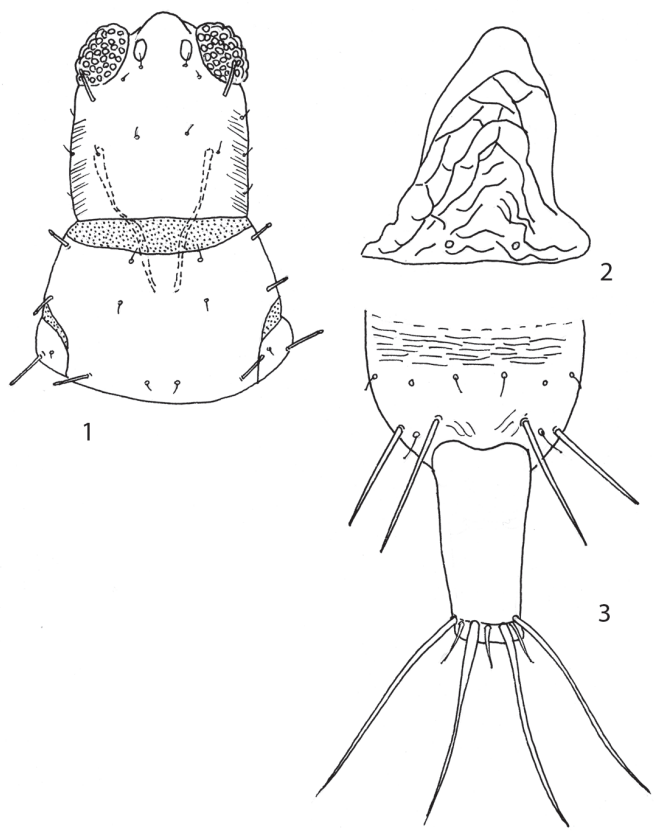

Figs. 1-3. Jironiella saidi sp. nov. 1.Cabeza y pronoto; 2. Pelta; 3. Segmentos abdominales IX y X.

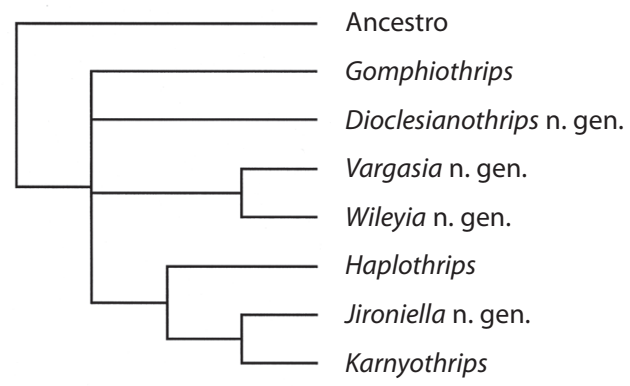

Fig. 4. Arbol filogenético del grupo Karnyothrips. $\mathrm{L}=14$, C.I. $=0.857$, R.I. $=0.800$. Mínimo de cambios posibles $=10$, Máximo de cambios posibles $=16$, Cambios registrados en el árbol=11.

prominencia. Lados de la cabeza ligeramente estriados y con tres setas cortas y agudas. Detrás de los ocelos se distinguen cuatro setas iguales a las anteriores. Estiletes maxilares muy apartados entre sí (Fig. 1) y no llegan más allá de la mitad de la cabeza. Segmento antenal III con 1 cono sensorial y segmento IV con 4 conos sensoriales. Setas mayores del pronoto poco desarrolladas. Metanoto con las setas medias agudas y con leve reticulación lateral. Pelta triangular (Fig. 2), más larga que ancha y con reticulación heterogénea, parte basal reticulada, parte distal sin reticulación, reticulación basal interna simple, posee dos sensilas campaniformes ubicadas en la parte basal y en el centro. Segmentos abdominales con 2 pares de setas retentorias de las alas. Seta B1 cerca de $2 / 3$ de la longitud del tubo y con el ápice truncado (Fig. 3). Segmento $\mathrm{X}$ del abdomen corto y ensanchado basalmente, no presenta reticulación.

Especie tipo: Jironiella saidi $\mathbf{s p .} \mathbf{n}$.

Etimología: dedicado al entomólogo médico Luis Fernando Jirón Porras, por sus frecuentes contribuciones a la entomología costarricense desde diversos ámbitos.

Comentarios: Este género se relaciona con Haplothrips en cuanto a la disposición de los conos sensoriales en los segmentos antenales III y IV, pero se diferencia de este porque carece de puente maxilar. Asimismo, se diferencia de los demás géneros de Phlaeothripidae por la forma de la pelta, la disposición de los estiletes maxilares, las setas del pronoto y la forma del tubo.

\section{Jironiella saidi sp. n. Retana \& Soto} (Figs. 1-3)

Material: Holotipo hembra macróptera. COSTA RICA: Puntarenas, Parrita (en zacatal detrás de la Escuela de Parrita). I-1994. (A.P. Retana-Salazar) en barrido de Cyperaceae. Paratipos: 17 hembras y 2 machos, todos recolectados con el holotipo.

Coloración: En general castaño amarillento, excepto los $2 / 3$ de las tibias anteriores y los segmentos antenales que son de coloración amarillo pálido.

Cabeza ancha y del doble de longitud que el segmento abdominal X. Segmento antenal IV es el de mayor longitud (45 $\mu \mathrm{m})$. Segmentos I-III y V-VIII entre 20-40 $\mu \mathrm{m}$. Setas postoculares de mayor longitud que las del pronoto y 
de ápices expandidos. Estiletes maxilares muy separados entre sí (Fig. 1) y no llegan más allá de la mitad de la cabeza.

Pronoto con las setas mayores de ápices expandidos, excepto las setas anteromarginales que son diminutas y agudas (la mitad de longitud que las setas anteroangulares).

Pelta triangular (Fig. 2), más larga que ancha, no posee reticulación en la parte apical. Segmento abdominal IX con la seta B1 cerca de $2 / 3$ de la longitud del tubo y de ápice truncado (Fig. 3). Seta B2 aguda y de mayor longitud que las otras, B3 de menor longitud y aguda. Segmento X del abdomen corto, la mitad de la longitud de la cabeza.

Medidas del holotipo (en micrómetros): Longitud total: 1775. Longitud de la cabeza: 180. Ancho de la cabeza a nivel de los ojos compuestos: 160. Setas postoculares 45 . Longitud de los antenómeros: I 32,5, II 40, III 40, IV 45, V 40, VI 35, VII 32,5, VIII 20. Longitud de las setas del pronoto: anteroangulares 25; anteromarginales 12.5 ; posteroangulares 35; epimeral 50; B1 65; B2 70 y B3 60. Setas terminales del abdomen 100. Segmento X: 90 .

Etimología: Dedicada a Said A. RetanaSalazar, por el constante y desinteresado apoyo que a los trabajos de investigación, y por su aporte al conocimiento de la matemática y la informática aplicados a la biología.

A continuación presentamos una clave separación de estos dos grupos genéricos, fundamentada en caracteres de valor evolutivo.

\section{Clave de separación del grupo Haplothrips y Karnyothrips}

1a) Puente maxilar presente (posiblemente reducido), estiletes maxilares separados a lo sumo una distancia similar a $1 / 3$ del ancho de la cabeza, estiletes maxilares llegando por encima de la mitad de la cabeza, con 4 conos sensoriales en el IV antenómero, con placas del praepectum desarrolladas . . . . . . . . . . . . . . . (Grupo Haplohtrips) 2

1b) Puente maxilar ausente, estiletes maxilares separados por una distancia superior a $1 / 3$ del ancho de la cabeza, estiletes maxilares llegando por debajo de la mitad de la cabeza, con 4 conos sensoriales en el IV antenómero, con placas del

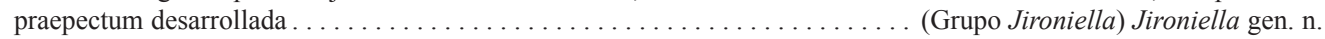

2a) Ausencia de diente terminal del tarso anterior de las hembras, especies en diversos hospederos $\ldots \ldots \ldots \ldots$

2b) Presencia de diente terminal del tarso anterior de las hembras en forma de uñas, especies asociadas a melastomatáceas

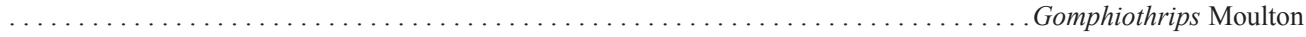

Género del grupo genérico Haplothrips, taxón conflictivo que fue considerado sinónimo de Treherniella por Priesner, y resucitado por Mound \& Marullo (1996) en virtud de la nueva especie mercedes. Ambas especies se hallan asociadas a la familia Melastomaceae, en todos sus estados de desarrollo, particularmente con respecto a los frutos secos. Se presenta en este género, dimorfismo sexual único en los phlaeothripinos, donde en el tergito IX la seta B1 es larga y aguda mientras que la B2 es corta y aguda.

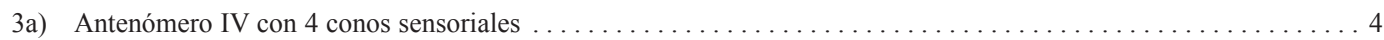

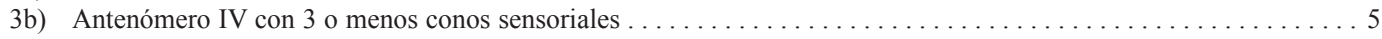

4a) Seta anal 1,5-2,0 veces la longitud del segmento $X$, setas am del pronoto reducidas, setas am reducidas con respecto a

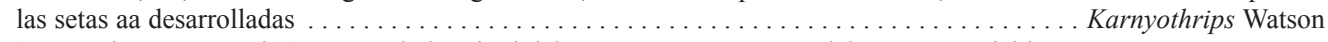

4b) Seta anal con menos de 1,5 veces la longitud del segmento $\mathrm{X}$, setas am del pronoto variables

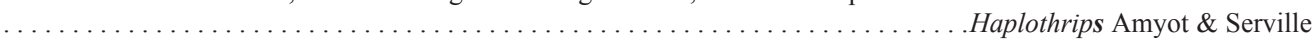

Género con más de 200 especies descritas. Posiblemente se trate de un conjunto de géneros mal agrupados por caracteres en su mayoría sin valor evolutivo. Es necesario un estudio cuidadoso del grupo para redefinirlo en función de sus caracteres fundamentales (Retana-Salazar y Retana-Salazar 2004). Actualmente se reconoce el subgénero Trybomiella en función del dimorfismo alar, sin embargo debe tratarse más bien de otro género y no de un subgénero.

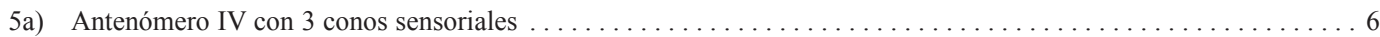

5b) Antenómero IV con 2 conos sensoriales, setas am desarrolladas subiguales a las setas aa desarrolladas

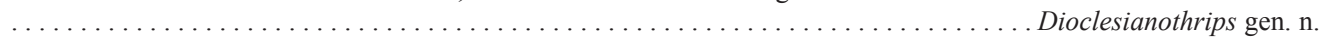

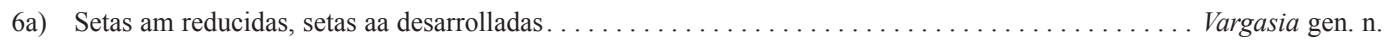

6b) Setas am desarrolladas subiguales a las setas aa $\ldots \ldots \ldots \ldots \ldots \ldots \ldots \ldots \ldots \ldots \ldots \ldots \ldots \ldots \ldots$ Willeia gen. $n$. 


\section{RESUMEN}

Se revisa el estado taxonómico del grupo Haplothrips y al mismo tiempo del grupo Karnyothrips, separándose este en varios nuevos géneros por considerarse parafilético; se describen los nuevos géneros Vargasia, Willeia y Aguilaria separados del antiguo Karnyothrips. Se describe un nuevo género de Thysanoptera-Phlaeothripidae procedente del Pacífico Central de Costa Rica. Este género se distingue por los estiletes maxilares muy apartados entre sí, carece de puente maxilar, la forma de la pelta y la seta B1 que es 2/3 la longitud del tubo. Se presenta el análisis crítico de este género y los posibles táxones emparentados, estableciendo un nuevo grupo a partir de las condiciones presentadas por Jironiella gen. n.

Palabras clave: Phlaeothripinae, Thysanoptera, nuevo género, Costa Rica.

\section{REFERENCIAS}

Ananthakrishnan, T.N. 1984. Bioecology of thrips. Indira, Oak Park, Michigan, EEUU. 233 p.

Lewis, T. 1973. Thrips: their biology, ecology and economic importance. Academic, Nueva York, Nueva York, EEUU. 349 p.

Goldarazena, A. \& L.A. Mound. 1998. The fauna of Tubulifera (Cl. Insecta: O. Thysanoptera) of Navarre (Northern Spain), with their habitat, host-plant and distribution data: An introduction. Est. Mus. Cienc. Nat. Alava 13: 185-200.

Mound, L.A., Heming, B.S. \& J.M. Palmer. 1980. Phylogenetic relationships between the families of recent Thysanoptera (Insecta). Zool. J. Linnean Soc.69: 111-141.

Mound, L.A. \& R. Marullo. 1996. The thrips of Central and South America: An Introduction (Insecta: Thysanoptera). Mem. Entomol. 487 p.

Mound, L.A. 1997. Biological diversity in Thrips as Crop Pests. T. Lewis CAB International, Londres. 740 p.

Mound, L.A. 2002. Thysanoptera biodiversity in the Neotropics. Rev. Biol. Trop. 50: 477-484.

Retana-Salazar, A.P. 1998. Una visión filogenética de Frankliniella (Thysanoptera: Thripidae). Rev. Biol. Trop. 46: 397-406.

Retana-Salazar, A.P. 2000. Revisión y filogenia del grupo Pseudothrips (Thysanoptera: Thripidae). Brenesia 54: 51-62.

Retana-Salazar, A.P. \& S.A. Retana-Salazar. 2004. Foro: Hacia una lógica simple en la determinación de grupos biológicos: la especie y los grupos supraespecíficos. Rev. Biol. Trop. 52: 19-26.

Retana-Salazar, A.P. \& G.A. Soto-Rodríguez. 2005. Una especie nueva de tisanóptero del género Frankliniella (grupo cephalica; Thysanoptera: Thripidae) de Costa Rica. Rev. Biol. Trop. 53: 191-194. 
